\title{
TERAPÉUTICA FÍLMICA: UNA POSIBILIDAD DE ASISTENCIA
}

\section{Film Therapeutics: a possibility of assistance}

Jeel MOYA-SALAZAR ${ }^{1,2}$; Betsy CAÑARI ${ }^{1}$; Lucia GOMEZ-SAENZ ${ }^{1}$; Alonso ZORRILLA ${ }^{1}$; Hans CONTRERAS-PU$\mathrm{LACHE}^{1}$

${ }^{1}$ Escuela de Medicina Humana, Facultad de Ciencias de la Salud, Universidad Privada Norbert Wiener, Lima (Perú). ${ }^{2}$ Hospital Nacional Docente Madre Niño San Bartolomé, Lima (Perú).

Autor para correspondencia: Hans Contreras Pulache

Correo electrónico: hans.contreras@uwiener.edu.pe

Recibido 26 de agosto de 2020

Aceptado 10 de septiembre de 2020

\section{Resumen:}

Existe una remarcada actividad del cine dentro de los procesos de formación médica promoviendo el desarrollo de múltiples habilidades inherentes. En este estudio, presentamos una propuesta de terapéutica fílmica en el contexto complementario de la asistencia médica actual. Revisamos series de antaño, emitidas alrededor de los 70`s, que constan de capítulos cortos ( $\sim 25$ minutos). Series como Candy Candy, Marco, o Heidi, tienen marcadas diferencias de las series contemporáneas, y el potencial de ser instrumentos terapéuticos en sí mismos debido a: i) presentar personajes concretos (un niño o una niña en particular, en un contexto específico), ii) la existencia de un relato argumentativo (que se sigue capítulo a capítulo, lo cual obliga a que el niño o niña recree longitudinalmente una historia), iii) el abordaje de problemas existenciales (la vida de los personajes los obligan a vivir situaciones límite, usualmente ligados a las condiciones históricas de su tiempo); y iv) la vivencia de experiencias de orden moral (que suponen una discusión sobre lo bueno y lo malo). Estos podrían vincular al niño y el padre, ya que cada capítulo representa una actividad de la vida diaria, eximiendo a estos de la transmisión masiva de series sin contenidos educativos (terapéuticos).

Palabras Clave: cine; terapia; series; neurología; asistencia parental. 


\begin{abstract}
:
There is a notable activity of the cinema within the medical training processes promoting the development of multiple inherent skills. In this study, we present a proposal for film therapy in the complementary context of current medical care. We review series of aforetime, broadcast around the 70`s, consisting of short chapters ( 25 minutes). Series like Candy Candy, Marco, or Heidi, have marked differences from modern series, and the potential to be therapeutic instruments in themselves due to: i) presenting specific characters (a boy or a girl in particular, in a specific context) , ii) the existence of an argumentative story (which is followed chapter by chapter, which forces the child to recreate a story longitudinally), iii) the approach to existential problems (the lives of the characters force them to live limit situations, usually linked to the historical conditions of their time); and iv) the experience of experiences of a moral nature (involving a discussion of good and bad). These could link the child and the father, since each chapter represents an activity of daily life, exempting them from the mass transmission of series without educational (therapeutic) content.

Keywords: cinema; therapy; series; neurology; parental assistance.
\end{abstract}

\section{Introducción}

Inútil sería resaltar el valor de la Revista Cine y Medicina. Inútil porque no necesita, desde aquí, que se le resalte: brilla sola. No precisa halagos ni felicitaciones. Sino agradecimientos. Para quienes vemos en el cine un gran artefacto como constructor de imaginarios, entonces, contar con este espacio específicamente ligado a la medicina, representa un lugar para habitar sin pasar la cuenta del tiempo.

Cierto es que el cine puede ayudar a los estudiantes de medicina, y a los profesionales de la medicina, a mejorar sus capacidades críticas, clínicas y de perspicacia ${ }^{1}$.Sin embargo, hay un hecho más, que poco aun ha sido explorado: el cine como herramienta terapéutica en sí. No ya el cine, para aprender algún aspecto de la medicina, sino el cine como medicina misma. El cine como herramienta médica en la lucha contra el dolor, el sufrimiento, y la muerte. El cine como instrumento terapéutico. En la medicina: una terapéutica fílmica.

Aquí, presentamos una propuesta de terapéutica fílmica en el contexto complementario de la asistencia médica actual, discutiendo las características de los programas animados, como estos pueden impactar sobre los pacientes, y discutiendo sus cambios progresivos hasta la actual era tecnológica.

\section{Nuestro campo de trabajo}

Vamos a precisar el campo desde el cual nos estamos orientando. En el tiempo moderno, uno de los grandes dilemas sociales es el que se vive al interior de las familias. Sobre todo, cuando existen infantes y niños; sobre todo cuando los padres y madres, sometidos a las inclemencias de la modernidad competitiva, deben pasar mucho tiempo en el entorno laboral, dejando en un segundo plano (aún con pesar) el ejercicio formativo de la paternidad y la maternidad.

Para quienes tratamos a familias con infantes y niños, nos salta siempre una evidencia práctica: muchos padres y madres conviven con los aparatos electrónicos de comunicación (por ejemplo: un televisor, un celular, una tablet, etcétera). A estos aparatos a veces, los padres/madres, les encuentran uso, pero muy mayoritariamente: se ven sometidos a sus embates. En el caso particular: casi todos, por no decir: todos, los infantes y niños, miran televisión, en algunos casos miran películas, pero más frecuentemente ven (una o varias horas al día) programas animados.

Se ha reportado previamente que niños de entre 2-5 años y 6-8 años consumen respectivamente $\sim 35$ y $\sim 28$ horas semanales de televisión ${ }^{2}$. $Y$ aunque los efectos negativos sean resaltantes, también se le ha atribuido a la televisión 
discutiblemente efectos positivos sobre el desarrollo de los niños usuarios ${ }^{3,4}$. Este impacto acumulativo de la televisión puede resumirse por ejemplo en el rol de la publicidad para que la ingesta de comida rápida se haya incrementado en niños 5 .

Al ver a un niño o niña comiendo en un restaurant frente a un celular, lo primero que sentimos es un error. Pero más precisamente debiéramos ver en esa situación la manifestación de una derrota: el aparato electrónico de comunicación ha ganado una batalla en la ejecución activa de la paternidad/ maternidad. Es decir, la tecnología, los aparatos tecnológicos como los teléfonos móviles, se han vuelto una prolongación orgánica, una prótesis perenne, una estructura casi intrínseca de nuestro organismo ${ }^{6}$. Pero aquí no queremos referirnos solo a los restaurantes, sino sobre todo a los hogares: donde la privacidad se diversifica hasta límites insospechables (y que solo se vislumbran con el ejercicio de una práctica clínica: ver, tratar pacientes). Las caricaturas se describen como uno de los modos más interesantes y entretenidos de transmisión de mensajes animados.

\section{Repetir Candy}

Por otra parte, para quienes ya llevamos algunas décadas sobre la tierra nos asalta un hecho singular: los programas animados contemporáneos distan mucho de los programas animados de antaño. Decimos «antaño» sin que esto nos traiga algún resabido del costumbrismo o del conservadurismo. Actualmente un programa animado se puede caracterizar como: una sesión de 30 o 60 minutos, salpicada con bastante propaganda comercial. ¿O es que son 30 o 60 minutos de propaganda salpicados con programas animados?

Como fuere: los programas animados contemporáneos gozan de la intensidad de la imagen, de la ahistoricidad del argumento, de la ficcionalidad extrema de los personajes (tendencia plena al caricaturismo), entre otras $\cos ^{7,8}{ }^{7,8}$. Todo esto hace que un infante o niño que mira un programa animado contemporáneo consuma propaganda, por un lado, y por otro se vea seducido por la erótica de los «dibujos animados». Usualmente, cada programa es un programa en sí mismo, cada sesión, mejor dicho: una sesión empieza y termina, y al otro día otra sesión empieza y termina. Lo único que pervive es el programa, pero no la trama argumental (la trama argumental se define en cada capítulo, en cada emisión, en cada sesión que empieza y que termina en un mar de propaganda).

La diferencia más esencial con los dibujos animados de antaño es que los personajes modernos son esencialmente abstractos (en contraste con los personajes antiguos que buscaban ser bastante concretos: piénsese en "Coraje, el Perro Cobarde (Courage the Cowardly Dog)" y en «Remi»-que tiene por compañera a una vaca-, en «Heidy»-que cohabita con los terneros-, en "Ángel»-que viaja por el mundo con un propósito: encontrar una flor de siete colores-). Por otra parte, las series animadas contemporáneas no precisan que el niño o niña sigan la secuencia de las sesiones. No hay secuencia, no hay serie, usualmente los capítulos son capítulos independientes: esto facilita que cualquier espectador se "enganche» con el programa animado en cualquier momento de su emisión, y no necesite: "conocer la historia» o «comprender el argumento narrativo».

Muchos padres y madres miran con sus hijos estos programas, los conocen, incluso se divierten y se ríen viéndolos. Sin embargo, poco o nada pueden luego conversar con sus hijos o hijas a propósito del mismo. Pueden pasar media o una hora siendo sacudidos por el mismo estímulo, pero luego que termina esta "exposición» nada queda que permita la relación dialógica de la paternidad/ maternidad.

\section{Series de antaño}

En lo posible la televisión fragmentalista actual, ha tratado uniendo esfuerzos con diversos intereses, en demoler lo poco que progresivamente aminoraba sobre la calidad mediática. Si 
se rescatan el imaginario de calidad de las series de antaño, podemos referir, como hacemos a continuación, las representaciones de cada serie y sus subestructuras, que nos atribuía afecto (y aun nos imputan) y una conexión establecida no solo con la serie transmitida, si no con el colectivo concernido a la trama de cada historia televisiva: Describiéremos a continuación, los componentes esenciales de cada serie que constituyen su argumento y su esencia, representadas a continuación en imágenes.

\section{Remi}

Remi el niño de nadie"/ lenakiko 1977/Osamu Dezaki.

En 1977 fue creado Remi, el niño de nadie por TMS Entertainment, basó su argumento en la novela francesa Sans Famille de H. Marlot, que tuvo más de una cincuentena de episodios que fueron de 25 minutos de duración cada uno. Esta serie fue emitida por Nippon Televisión para Japón (periodo de transmisión: 2 de octubre de 1977 - 1 de octubre de 1978).

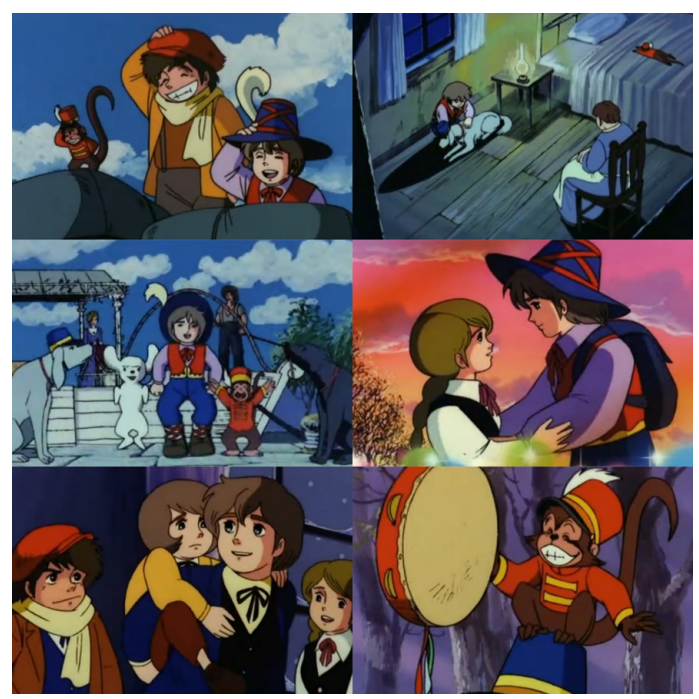

Este anime desarrolla la historia de Remi Milligan, niño inglés que fue secuestrado y luego impuesto a los Barberin en Chavanon. El padrastro el Señor Vitalis, músico callejero con antecedentes de fracaso en la ópera, posibilita que Remi trabaje como ayudante y músico. Durante este trabajo, Remi desarrolla mucho aprecio de los animales que usan en sus presentaciones, como Capi (un perro) y Corazón Alegre (un mono). El invierno ocasiona como parte de la trama un papel importante ya que ocasiona la muerte del Señor Vitalis y Corazón Alegre debido a la tormenta de nieve y la hipotermia, respectivamente. Otro hecho trascendental es Mattia, un niño italiano, músico y rebelde que contrapone a Remi y que lo acompaña en nuevas aventuras como músicos. La principal es la búsqueda de su madre en Inglaterra.

El desarrollo profesional se desarrolla hacia el final del anime. Esta serie impresionista resalta el dramatismo europeo y expone al espectador a una seguidilla de acontecimientos con valor humano, a los que Remi intenta sobreponerse en búsqueda de su bienestar.

\section{Heidi}

Heidi, la niña de los Alpes/Arupusu no Shōjo Heidi (1974) / Isao Takahata

El estudio de animación japonés «Zuiyo Eizo» (actualmente, Nippon Animation) y, en conjunto con la cadena alemana Zweites Deutsches Fernsehen, estrenó el 5 de enero de 1974 la serie infantil: «Heidi, la niña de los Alpes». La historia es ambientada en el siglo XIX, mucho antes de la primera guerra mundial (1914) e incluso antes de la guerra franco-prusiana (1870-1871); es inspirada en el libro homónimo de Johanna Spyri (18271901), obra basada en su niñez.

Figura 1. Infograma sobre Remi, el niño de nadie. 


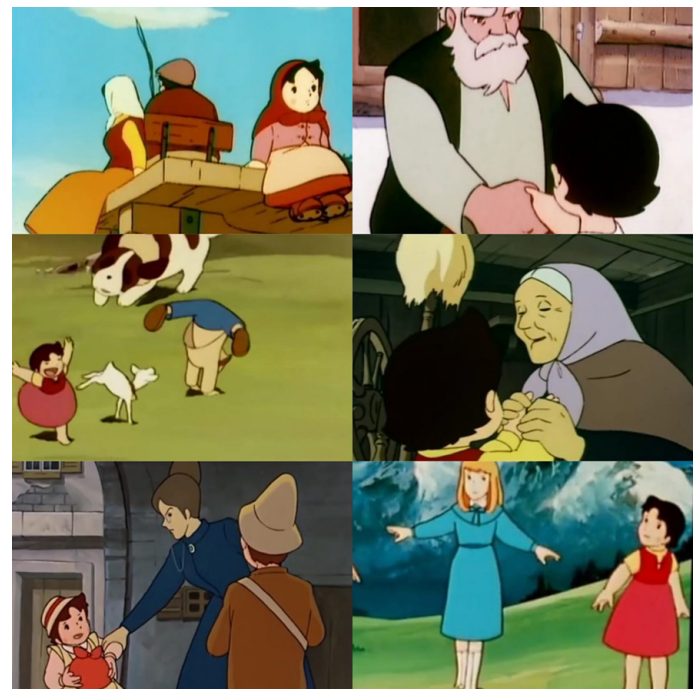

Figura 2. Infograma sobre Heidi, la niña de los Alpes.

\section{Anne}

Ana de las Tejas verdes/ Akage no An (1979) / Isao Takahata.

Ana la de Tejas Verdes, Anne la de Tejados Verdes o Ana de las Tejas Verdes (en inglés Anne of Green Gables) es un libro escrito por la canadiense Lucy Maud Montgomery y publicado por primera vez en 1908. La obra narra la vida de Anne Shirley, una niña huérfana que gracias a su carácter imaginativo y despierto logra encandilar a todos los habitantes de Avonlea, el pequeño pueblo pesquero ficticio en la Isla del Príncipe Eduardo donde se desarrolla la historia a principios del siglo XX.

El resto del libro relata su educación en la escuela, donde pronto sobresale en sus estudios, sus ambiciones literarias y sus amistades con otras niñas como Diana Barry (su mejor amiga), Jane Andrew o Ruby Gillis, y su manifiesta rivalidad con Gilbert Blythe, un chico tres años mayor que Ana que osó hacer una broma acerca de su pelo y adquirió a cambio el odio de la pelirroja, aunque él le pidiera perdón repetidamente. El libro también sigue las desventuras de Ana en la pacífica Avonlea. En las que se incluyen sus juegos con su grupo de amigas (Diana, Jane y Ruby), sus rivalidades con las hermanas Pye (Gertie y Josie) sus errores domésticos, como teñirse el pelo de verde, entre otros.

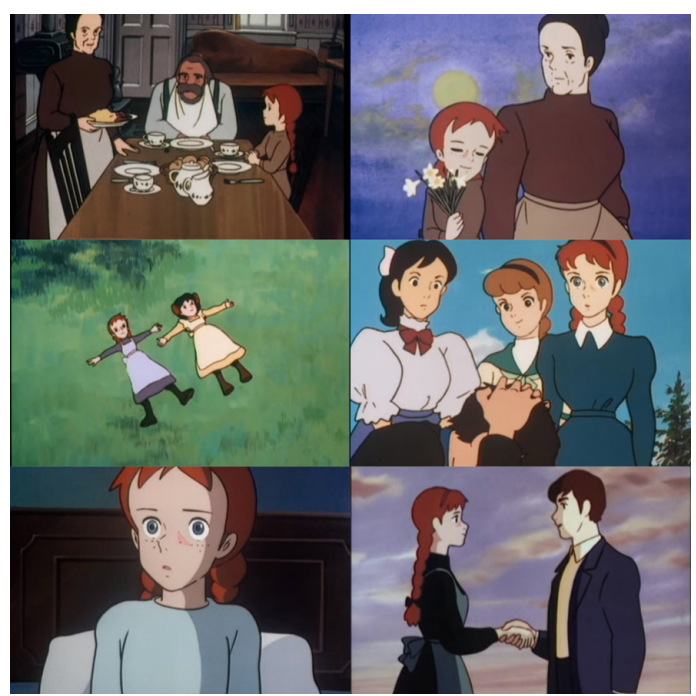

Figura 3. Infograma sobre Ana de las Tejas Verdes.

Por lo mismo, ni bien nos sumergimos en la historia, nos vemos envueltos en un contexto en donde no es posible percibir tecnología industrial alguna. $Y$ de esta manera abordamos a una fantástica serie que, a su vez, es tan ajena a lo fantástico, pues los protagonistas nos encarnan a medida en que nos enseñan cómo una persona puede influir tanto en la vida de otra. Así, Heidi expone la alegría, la inocencia, la libertad. Clara Sesemann representa el esfuerzo, lo indefenso. El abuelo, Tobías Hessen, la soledad. A lo largo de este marco de guerra y unión, los personajes exteriorizan diversos tipos de convivencia, la gratitud, la diversión, lo irónico y lo detallista; y en contraste, el egoísmo, lo inesperado, la tristeza y la chocante desesperación al verse privados de su 
fuente o concepto de felicidad. Los 52 gloriosos episodios se estrenaron en España con un doblaje peninsular en 1975 y en Latinoamérica en 1978 con un doblaje mexicano.

\section{Marco}

Marco, de los Apeninos a los Andes/Haha o Tazunete Sanzenri (1976)/ Isao Takahata

Marco, de los Apeninos a los Andes es un breve relato de incluido en la novela Corazón por Edmundo de Amicis publicada en el año 1886, que describe la inclemente visión sobre la emigración italiana hacia América, entre el siglo XIX e inicios del siglo XX.

Este anime de 52 episodios fue emitido por primera vez en 1976 en Japón. La serie narra la vida de una familia de Génova, Italia, conformada por una madre con dos hijos, el menor y más pequeño llamado Marco. La historia comienza cuando la madre se ve obligada viajar a la Argentina para conseguir un trabajo y brindarles solvencia económica y una mejor calidad de vida a sus dos hijos que residen en Génova. Los hermanos reciben correspondencia de la madre, desde América, hasta que gradualmente no tuvieron noticias de ella en un periodo de dos años.

Por este devenir, Marco, de 13 años decide afrontar un largo y complicado viaje desde Italia hacia Argentina con el propósito de hallar a su madre. Marco, acompañado de Pepino su mono, inicia su viaje que incluye singulares experiencias, el descubrimiento de personajes con diferentes características demostrando las divergencias entre costumbres y realidades de cada estación del viaje. Luego en Argentina, recorre varias ciudades que incluyen Buenos Aires, Córdoba, y Tucumán para encontrar a su madre.

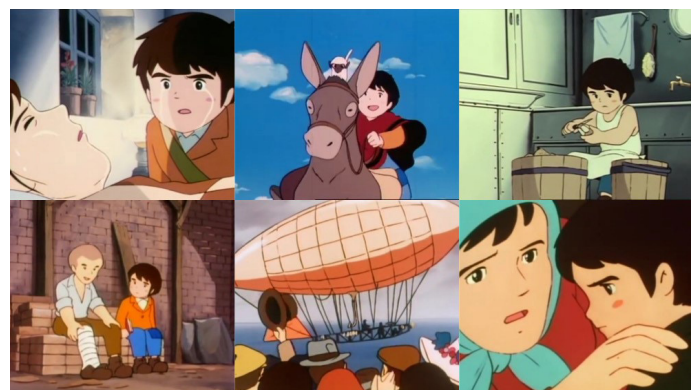

Figura 4. Infograma sobre Marco, de los Apeninos a los Andes

\section{Candy, Candy}

Candy Candy/ Hiroshi Shidara (1975)

Hace más de cuatro décadas, el estudio Japones Toei Animation estreno Candy Candy, emitido por primera vez en octubre de 1976, siendo uno de los principales y Cosmopolitan animes que durante décadas ha conquistado al público infantil con su centenar de episodios de 25 minutos cada uno.

Este anime narra en 115 capítulos la historia de una niña huérfana llamada Candy, hallada un día de nieve a principios del siglo XX. Es en el orfanato Ponys home cercano al Lago Michigan, donde crece junto a su gran amiga Annie, huérfana y hallada el mismo día que ella. Se vuelven inseparables amigas hasta el día en que deciden adoptar a Candy, que sin dudar y recordando la promesa de inseparabilidad con Annie se rehúsa a ser adoptada. Sin embargo, los padres adoptivos ante esta situación deciden adoptar a Annie, que a diferencia de Candy acepta sin dudar y se marcha del orfanato. A sus 12 años de edad Candy es adoptada por la familia Leagan para que acompañe a sus dos hijos Elisa y Neil, quienes la trataron muy mal, hasta convertirla en sirvienta, incluso la acusaron de un robo e intentaron enviarla a una granja de su familia a México a trabajar, pero fue salvada por William Adley quien fue el padre adoptivo de Candy. Candy convive con una mascota 
(Klin) que sostiene su tristeza antes el iryvenir que la vida le ofrece. Aunque la vida de Candy se desarrolla entre la miseria y el advenimiento de problemas existenciales, a su vez está rodeada de personas que la estimaban mucho como como Archie, Anthony, o Terry.

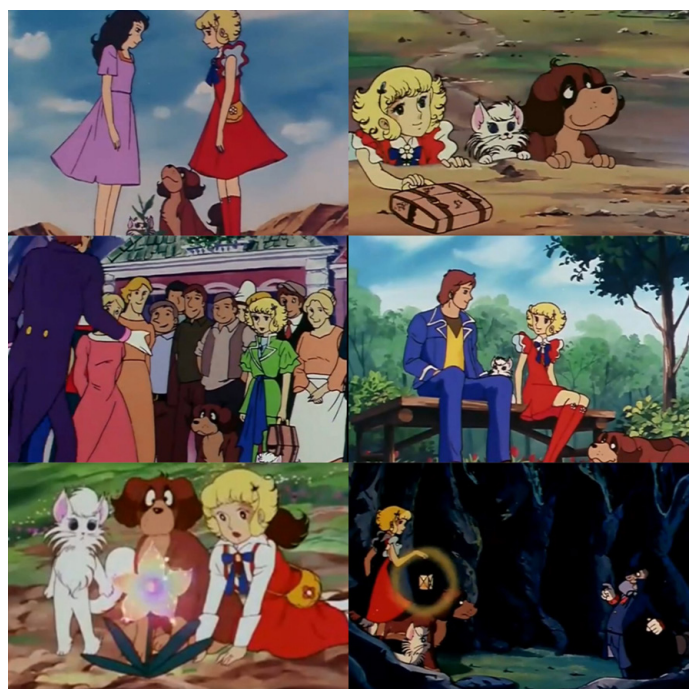

Figura 5. Infograma sobre Candy Candy.

\section{La novicia rebelde}

Torappu ikka monogatari/ Kōzō Kusuba, Kōzō Kuzuha (1991)

En el año 1991 fue emitida por primera vez en idioma Japones La novicia rebelde. Una serie creada por Nipón Animation. Conocida también como Sonrisas y lágrimas, esta serie narra la historia de una niña llamada María de 18 años que desea ser monja y dedica todos sus años de vida a Dios, sin embargo, su personalidad energética y divertida es advertida por la madre superiora. Por ello, es llevada por un tiempo a la casa de Barón Von Trapp como institutriz de sus hijos. Este señor viudo y en esa casa se había contratado 25 institutrices siendo todas rechazadas por sus hijos, sin embargo, María con su alegría, poesía y canción logra ganarse el respeto y corazón de los integrantes de la familia. Su intento de comprometerse con Barón Von Trapp es amenazado por la Segunda Guerra Mundial y el control tomado por los nazis genera que la familia migre a Suiza. Los 40 Episodios de esta serie, inician con el capítulo Quiero ser monja y el terminan con el último Adiós Patria.

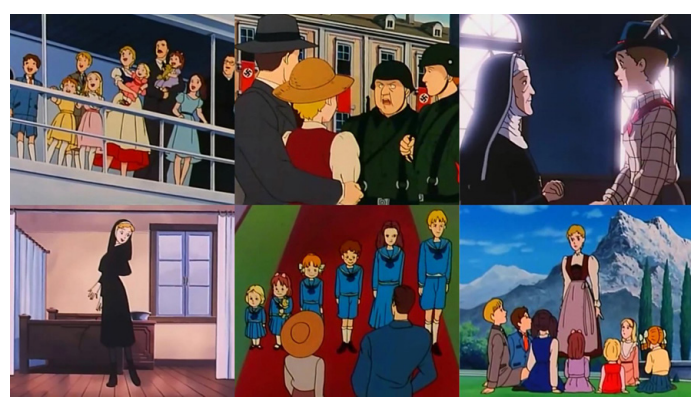

Figura 6. Infograma sobre la novicia rebelde

\section{El cine animado de antaño}

En este aspecto vamos a precisar que el cine (en particular las series animadas de la década de los 70 s y 80 s, a las cuales llamamos "de antaño», casi unánimemente elaboradas como obras primeras de los que luego (hasta la actualidad) se convertirían en los grandes representantes del cine animado japonés. Por ejemplo: Isao Takahata, que luego se convirtió en uno de los emblemas del studio Ghibli (Tokio, Japón), junto con Hayao Miyasaki. Lo que estamos diciendo es que estas series (por ejemplo: «Heidi, la niña de los Alpes», "Marco», "La Novicia Rebelde», "Candy Candy», "Remi», "Annie, la de las Tejas Verdes», entre otras) representan una herramienta educativa (que puede facilitar más llanamente los roles de la paternidad y la maternidad) esencialmente, porque a diferencia de las series animadas contemporáneas, muestran los siguientes 4 hechos trascendentales, sin decir que estos sean los únicos: 
- Personajes concretos (un niño o una niña en particular, en un contexto específico);

- Existe un relato argumentativo (hay una trama que debe seguirse capítulo a capítulo, lo cual obliga a que el niño o niña recree longitudinalmente una historia);

- Se abordan problemas existenciales (la vida de los personajes -concretos- los obligan a vivir situaciones límite, usualmente ligados a las condiciones históricas de su tiempo); y

- Usualmente el personaje (un niño o niña) vive una serie de experiencias de orden moral (que suponen una discusión sobre lo bueno y lo malo).

Consideramos que estos elementos (sin necesidad de afirmar que sean los únicos que agotan el tema) permiten diferenciar claramente la experiencia de un niño o niña que mira, por ejemplo: "Marco» y que por lo mismo debe recrear en su "mente» (mejor diríamos: en su conciencia) toda una historia llena de vicisitudes trascendentales, y la experiencia del mismo niño o niña que mira, por ejemplo: "La Patrulla Canina», "Rick y Morty» o "Gravity Falls». Pareciera que la evolución de las series animadas "infantiles» ha seguido un derrotero: sacrificar la aspiración de reflejar una problemática real por proporcionar (a la propaganda) una sesión de "aventura», diversión, risa, burla, caricatura, y superficialidad.

¿Es que realmente se piensa que un niño o niña viendo "Gravity Falls» logra aprender sobre los «Misterios en Hispanoamérica» (recordemos que el título completo de la serie es: «Gravity Falls: ¿Un Verano de Misterios en Hispanoamérica»)?

Lo peor no queda aquí, para quienes tenemos pacientes que son personas concretas: nos salta que las series animadas contemporáneas son un espacio esencialmente para ser visto por niños y niñas y que puede ser también vistos por los padres/madres, pero que luego de verlos: no se promueve en nada la construcción de la paterni$\mathrm{dad} / \mathrm{maternidad}$, pese a que se ha destacado ser conmovedoras e inteligentes ${ }^{9,10}$. Atención, con esto no queremos ser conservadores, no queremos afirmar "las series antiguas eran mejores», no queremos criticar a la televisión, ni siquiera estamos diciendo que el cine debe ser utilitario ni que la paternidad/maternidad se vean sometidas a la televisión y a otros aparatos electrónicos de comunicación (alguna aplicación o el YouTube en un celular cualquiera, por ejemplo).

Solo estamos resaltando que a la hora de crear paternidad/maternidad se precisan cuando menos dos sujetos: uno en la posición de la adultez y otro en la posición de la niñez, y que el tiempo moderno de la globalización postcapitalista, o como se dice: del tardocapitalismo, ha sometido el tiempo de las personas que en tanto adultos transitan la paternidad/maternidad. $Y$ que cuando dicho tiempo logra finalmente establecerse: es parasitado por un aparato de televisión, o más modernamente, por un simple celular o Tablet. Que se diga por todas partes que un niño o niña no debe tener una Tablet y que se afirme categóricamente esta propuesta no deja de ser una aspiración, lo que en el mundo hay es niños y niñas que no necesitan tener un celular o Tablet o un televisor: si ellos no lo tienen, los padres/madres sí (ellos se esfuerzan en ello, es un signo de reputación tener un buen celular, de éxito competitivo, etcétera).

No vamos a caer en la retórica de repetir: el celular y la Tablet son «malos», no vamos a perder el tiempo así, más específicamente queremos resaltar que en los años 70 s en adelante se elaboraron unas series animadas, radicalmente diferentes a las series animadas contemporáneas. Frente al exceso de la estimulación visual y auditiva de la animación contemporánea, se cuenta con imágenes clásicas (inspiradas en el manga desarrollado por Tezuka), con obras de profundo carácter humano (demasiado humano), y que pueden promover la conversación y diálogo entre padres/madres e hijos (niños y niñas), más ahora que existe una cultura de promoción de la cultura japonesa y del anime ${ }^{11}$. 
Sin embargo, la actual sociedad informática está soslayando las relaciones interpersonales, que se reflejan principalmente en el seno familiar. La extraversión permanente con la tecnología está irrumpiendo dramáticamente en el entorno social, y esto, está promoviendo que los niños estén más dispuestos hacia los dispositivos tecnológicos, dificultando la interacción con otros sujetos. Los padres/madres deben de acometer antes esta contingencia con herramientas periféricas a la tecnología o la tecnología con otro enfoque de uso a priori. Recientemente se ha demostrado mayores probabilidades de que los padres interaccionen con sus hijos menores de 12 años a través de los animes $^{12}$. Entonces, los animes representan una estrategia novedosa de interacción, en un sentido mayor que solo la contemplación de caricaturas con fines de educación de valores y la sociedad, como se ha señalado previamente ${ }^{13}$.

En este sentido, el cine, en particular: las series animadas son instrumentos terapéuticos en sí mismos. No solo sirven para aprender medicina, sino sirven para hacer con ellos lo que la medicina, creemos, pretende desde su seno más profundo: reelaborar el sufrimiento.

\section{Agradecimientos}

Agradecemos la participación de Anie SorianoAbal y Samanta Picón de la Universidad Norbert Wiener, a Matilda Contreras del Centro de Documentación Pedro Ortiz Cabanillas, y a Andrea Mija de la Facultad de Educación de la Universidad Nacional Mayor de San Marcos por su apoyo en la estructuración grafica del estudio.

\section{Referencias}

1. Kadivar M, Mafinejad MK, Bazzaz JT, Mirzazadeh A, Jannate Z. Cinemedicine: Using movies to improve students' understanding of psychosocial aspects of medicine. Ann Med Surg (Lond). 2018; 28: 23-7.
2. McDonough P. TV viewing among kids at an eightyear high. 26 de octubre de 2016. New York: Media, The Nielsen Company American.

3. Kukreja J. Cartoons Cast an Eternal Impact on Personalities: Effects of Cartoons on Children. In: Sarı G (Ed.), Handbook of Research on Children's Consumption of Digital Media. Pennsylvania: ISI Global Disseminator of Knowledge; 2019.

4. Rai S, Waskel B, Sakalle S, Dixit S, Mahore R. Effects of cartoon programs on behavioural, habitual and communicative changes in children. Int J Community Med Public Health. 2016;3(6):1375-78.

5. Dalton MA, Longacre MR, Drake KM, Cleveland LP, Harris JL, Hendricks K, et al. Child-targeted fast-food television advertising exposure is linked with fast-food intake among pre-school children. Public Health Nutr. 2017; 20(9): 1548-56.

6. Denegri MA. El dios de la prótesis. 06 de agosto de 2017. Lima: Libros. Diario EL Comercio. [Consultado: 04/07/2020].

7. Klein NM. Seven Minutes: The Life and Death of the American Animated Cartoon. New York: Verso; 1993.

8. McGowan D. Animated Personalities: Cartoon Characters and Stardom in American. University of Texas Press; 2019.

9. Wilford D. 'Gravity Falls' Review: The Shack Is Back, And Scarier Than Ever Before. HUFFPOST. 23 de octubre de 2014. [Consultado: 04/09/2020].

10. Blum M. Gravity Falls rises to the level of Disney Channel's best. WIRED [internet]. 28 de junio de 2012. [Consultado: 04/05/2020].

11. Agyeiwaah E, Suntikul W, Carmen LYS. «Cool Japan»: Anime, soft power and Hong Kong generation $Y$ travel to Japan. J China Tourism Res. 2018; 1-22.

12. Yamamura $E$. The effect of young children on their parents' anime-viewing habits: evidence from Japanese microdata. J Cult Econ. 2014; 38: 331.

13. Akinyi KMC. The necessity for parents to watch animated cartoons with children aged seven to eleven years. J Educ Pract. 2018; 2(1):42-52. 


\begin{tabular}{|c|c|}
\hline (-) & $\begin{array}{l}\text { Jeel Moya-Salazar. Tecnólogo Médico en la especialidad de Laboratorio } \\
\text { Clínico y Anatomía Patológica, es egresado de la Maestría en Ciencias con } \\
\text { Mención en Bioquímica y Biología Molecular, y Maestro de la Maestría en } \\
\text { Salud Publica por la Universidad Privada San Juan Bautista. Ha colaborado } \\
\text { en proyectos de investigación en la Samara University (Rusia), Stanford } \\
\text { University (US), y Universidad de Buenos Aires (Argentina). Es investigador } \\
\text { RENACYT en la categoría Carlos Monge III - concytec y es investigador } \\
\text { estudiantil del equipo de investigación de estudiantes de Medicina en } \\
\text { Neurociencias aplicadas. Es miembro de ALLBIOTECH. }\end{array}$ \\
\hline & $\begin{array}{l}\text { Betsy Cañari Ccoica. Estudiante de cuarto año de la facultad de Medicina } \\
\text { Humana de la Universidad Norbert Wiener, Lima Perú. Miembro del equipo } \\
\text { de investigación de estudiantes de Medicina en Neurociencias aplicadas. }\end{array}$ \\
\hline & $\begin{array}{l}\text { Lucia del Carmen Beatriz Gomez Saenz. Estudiante de tercer año de la } \\
\text { facultad de Medicina Humana. Egresada en Advanced English del Insti- } \\
\text { tuto Cultural Peruano Norteamericano (ICPNA) en el 2016. Certificada en } \\
\text { Methodology for EFL teachers en el año 2017. }\end{array}$ \\
\hline & $\begin{array}{l}\text { Alonso Jean Pierre Zorrilla Chávez. Estudiante del segundo año de la facul- } \\
\text { tad de Medicina Humana. Actualmente representante de televentas para } \\
\text { movistar Argentina en Konecta. }\end{array}$ \\
\hline & $\begin{array}{l}\text { Hans Contreras-Pulache. Es Médico Cirujano por la Universidad Nacional } \\
\text { Mayor de San Marcos. Se ha especializado en epidemiología, salud pública, } \\
\text { gerencia social y gestión pública. Se ha hecho responsable, desde el 2011, } \\
\text { de toda la obra académica de Pedro Ortiz Cabanillas. Como parte de este } \\
\text { trabajo editorial ha publicado: «La explicación científica del hombre» (2013), } \\
\text { «El sistema de la personalidad» (2016), «Psicobiología social» (2017) y «La } \\
\text { explicación informacional» (2019). Su obra personal ha principiado con } \\
\text { «Neurología Fílmica» (2016). }\end{array}$ \\
\hline
\end{tabular}

